\title{
Silica-coated iron-oxide nanoparticles doped with Gd(III) complexes as potential double contrast agents for magnetic resonance imaging at different field strengths
}

\author{
ALEXEY STEPANOV ${ }^{\mathrm{a}, *}$, SVETLANA FEDORENKO ${ }^{\mathrm{a}}$, RUSTEM AMIROV ${ }^{\mathrm{b}}$, \\ IREK NIZAMEEV $^{\mathrm{c}}$, KIRILL KHOLIN ${ }^{\mathrm{a}}$, ALEXANDRA VOLOSHINA ${ }^{\mathrm{a}}$, \\ ANASTASIYA SAPUNOVA ${ }^{\mathrm{a}}$, RAFAEL MENDES ${ }^{\mathrm{f}}$, MARK RÜMMELI $^{\mathrm{d}, \mathrm{e}, \mathrm{f}}$, \\ THOMAS GEMMING $^{\mathrm{f}}$, ASIYA MUSTAFINA ${ }^{\mathrm{a}}$ and BORIS ODINTSOV ${ }^{\mathrm{g}, \mathrm{h}}$ \\ ${ }^{a}$ Arbuzov Institute of Organic and Physical Chemistry, FRC Kazan Scientific Center of RAS, Arbuzov Str., 8, \\ Kazan, Russia 420088 \\ ${ }^{\mathrm{b}}$ Kazan (Volga region) Federal University, Kremlyovskaya Str., 18, Kazan, Russia 420008 \\ ${ }^{\mathrm{c}}$ Kazan National Research Technological University, Kazan, Russia 420015 \\ ${ }^{\mathrm{d}}$ Soochow Institute for Energy and Materials Innovations, College of Physics, Optoelectronics and Energy \\ \& Collaborative Innovation Center of Suzhou Nano Science and Technology \& Suzhou Key Laboratory for \\ Advanced Carbon Materials and Wearable Energy Technologies, Soochow University, Suzhou 215006, China \\ ${ }^{\mathrm{e}}$ Centre of Polymer and Carbon Materials, Polish Academy of Sciences, M. Curie-Sklodowskiej 34, 41-819 \\ Zabrze, Poland \\ ${ }^{\mathrm{f}}$ IFW Dresden, Helmholtz Strasse 20, 01069 Dresden, Germany \\ ${ }^{\mathrm{g}}$ Biomedical Imaging Center of the Beckman Institute for Advanced Science and Technology, University of \\ Illinois, Urbana-Champaign IL 61801, USA \\ ${ }^{\mathrm{h}}$ Department of Bioengineering, University of Illinois, Urbana-Champaign IL 61801, USA \\ E-mail: aleksestepanov@yandex.ru
}

MS received 16 May 2018; revised 24 June 2018; accepted 25 June 2018; published online 27 August 2018

\begin{abstract}
In the present work, we have prepared the $\sim 10 \mathrm{~nm}$ superparamagnetic iron-oxide nanoparticles by means of high-temperature decomposition of iron oleate precursor. Then they were coated with silica shell to impart water-solubility and an ability to accommodate paramagnetic Gd(III)-based complexes inside silica coating. All of the prepared nanoparticles form stable in time aqueous dispersions and show good negative or/and positive contrasting effect at different magnetic field strengths $(0.47,1.41,14.1 \mathrm{~T})$. It has been also exemplified that the incorporation of [Gd(TCAS)] complexes into silica shell triggers a significant increase of the transverse relaxivity of the core-shell nanoparticles. The correlation between relaxometric properties and morphology of the obtained nanoparticles was revealed. The non-toxicity of the obtained nanoparticles along with their ability to shorten both transverse and longitudinal relaxation rates of water protons make them good candidates for their use as dual-mode contrast agents in MRI.
\end{abstract}

Keywords. Double contrast agents; silica nanoparticles; longitudinal relaxivity; transverse relaxivity.

\section{Introduction}

Iron-oxide nanoparticles along with $\mathrm{Gd}(\mathrm{III})$ chelates have attracted significant attention of researches in recent decades as potential contrast agents in MRI due to their ability to shorten transverse and longitudinal relaxation times of water protons, respectively, allowing better interpretation of different tissue lesions and abnormalities in a non-invasive manner. ${ }^{1-3}$ The commonly applied single-mode imaging agents, which are based either on iron-oxides or Gd(III) chelates, suffer from some drawbacks. For instance, Gd(III) chelates raise the toxicity and high mobility issues, whereas the

\footnotetext{
*For correspondence

Electronic supplementary material: The online version of this article (https://doi.org/10.1007/s12039-018-1527-z) contains supplementary material, which is available to authorized users.
} 
strong magnetic field of iron-oxide nanoparticles may lead to magnetic susceptibility artefacts and quench the $\mathrm{T}_{1}$ signals of $\mathrm{Gd}(\mathrm{III})$ chelates. In addition, iron-oxide nanoparticles may lead to poor contrast, aggregation and accumulation in the tissues and organs. ${ }^{4,5}$ To overcome the above-mentioned difficulties of single-mode contrast agents, the development of nanoparticulate probes combining both superparamagnetic and paramagnetic units would be very desirable for more accurate imaging. The general approach proposed by the authors includes the core-shell structure where iron-oxide nanoparticles are encapsulated into silica matrix which is doped with Gd(III)-based paramagnetic material and 'covalent approach' in which Gd chelates are covalently attached to the silica shell of the iron-oxides. ${ }^{67}$ In particular, Cheon et al., has reported in vivo dual contrasting by means of iron-manganese-oxide nanoparticles coated with $\mathrm{Gd}_{2} \mathrm{O}_{3}$-doped silica. By tuning the length of the silica separating layer between the superparamagnetic core and paramagnetic outer shell the authors have managed to obtain a very promising transverse and longitudinal relaxivities (274 and $33.1 \mathrm{mM}^{-1} \mathrm{~s}^{-1}$ at $4.7 \mathrm{~T}$, respectively) along with high diagnostic accuracy. ${ }^{4}$ The similar nanoparticulate structure was proposed by Peng et al. ${ }^{8}$ These works demonstrate the 'core-shell' approach in the design of novel double contrast agents.

As far as the 'covalent approach' is concerned, it is worth to mention the work of Zapotoczny et al., who has published superparamagnetic chitosan-protected ironoxide nanoparticles covalently bound with Gd-DTPA complexes. ${ }^{7}$ The obtained nanoparticles showed very high $\mathrm{R}_{2}$ and $\mathrm{R}_{1}\left(375.5\right.$ and $53.7 \mathrm{mM}^{-1} \mathrm{~s}^{-1}$ at $15 \mathrm{MHz}$, respectively, $\left.R_{2} / R_{1}=6.99\right)$. The papers ${ }^{9-11}$ have also reported similar 'covalent approaches' for producing the nanoparticles with promising transverse and longitudinal relaxivities.

In the present paper, we have coated $10 \mathrm{~nm} \mathrm{Fe}{ }_{3} \mathrm{O}_{4}$ cores with silica to ensure water-solubility and biocompatibility. The resulting nanoparticles demonstrated high transverse relaxivity of $134.74 \pm 1.78 \mathrm{mM}^{-1} \mathrm{~s}^{-1}$ making them a promising single-mode negative contrast agent. We have also prepared the iron-oxide nanoparticles coated with paramagnetic [Gd(TCAS)]-doped silica shell. Our aim was to test the single and double core-shell nanoparticles as prospective negative and negative/positive (double) contrast agents at different magnetic field strengths and to reveal the possible correlation between relaxometric behaviour and morphology of the nanoparticles. As expected, the obtained nanoparticles showed high transverse and longitudinal relaxivity as well as negligible cytotoxicity making them a promising basis for further use as double contrast agents in MRI.

\section{Experimental}

\subsection{Materials}

Commercial chemicals tetraethyl orthosilicate (TEOS) 98\%, ammonium hydroxide (28-30\%), $n$-heptanol 98\%, cyclohexane $99 \%, \mathrm{Gd}\left(\mathrm{NO}_{3}\right)_{3} \cdot 6 \mathrm{H}_{2} \mathrm{O}(99.90 \%)$, hexane $(97 \%)$, acetone (99\%), chloroform (99.8\%) were purchased from Acros Organics. Triton X-100 (98\%), NaOH, oleic acid (99\%), octadecane, sodium oleate were purchased from SigmaAldrich. Chloroform was purified as follows: a certain volume of $\mathrm{CHCl}_{3}$ was washed several times with water and concentrated $\mathrm{H}_{2} \mathrm{SO}_{4}$, dried over $\mathrm{K}_{2} \mathrm{CO}_{3}$ and finally, was distilled at atmospheric pressure. Ethanol was purified by distillation under atmospheric pressure, whereas TEOS was distilled under reduced pressure. All other chemicals were used as received without further purification.

The standard nutrient medium 'Igla' was obtained from Moscow Institute of poliomyelitis and viral encephalitis named after M. P. Chumakov.

\section{1a Synthesis of iron oleate complex and}

p-sulfonatothiacalix [4]arene: Iron oleate was synthesized according to published method. ${ }^{12} p$-sulfonatothiacalix [4]arene (TCAS) was prepared by means of the well-known literature procedure. ${ }^{13}$

\section{1b Synthesis of $10 \mathrm{~nm}$ iron-oxide nanoparticles:}

Iron-oxide nanoparticles were synthesized according to wellknown literature method. ${ }^{12-14}$ Firstly, $3 \mathrm{~g}$ of iron oleate (III) was dissolved in $10.8 \mathrm{~mL}$ of octadecane with $1 \mathrm{~mL}$ of oleic acid and heated up to $318^{\circ} \mathrm{C}$ (heating rate $3.3^{\circ} \mathrm{C} / \mathrm{min}$ ) for 30 min under argon in a reflux system. Then, the resulting solution containing iron oxide crystals was cooled down to room temperature and a mixture of hexane and acetone (volume ratio 1:4) was added to precipitate the crystals. Next, the nanoparticles were separated by centrifugation (20 min, $12,500 \mathrm{rpm}$ ) and washed 7 times with chloroform to remove excess of oleic acid and organic solvents remaining in the sample. The obtained nanoparticles were dried in vacuum at $90^{\circ} \mathrm{C}$ for $1.5 \mathrm{~h}$ and characterized.

2.1c Synthesis of silica-coated iron-oxide nanoparticles (SNs-I): $0.016 \mathrm{~g}$ of the synthesized oleate-coated iron-oxide nanoparticles were dispersed in $5 \mathrm{~mL}$ of cyclohexane using ultrasound water bath for $60 \mathrm{~min}$. Then, the obtained dispersion was added dropwise using a syringe pump (adding rate $1 \mathrm{~mL} / \mathrm{min}$ ) to the mixture of $2.38 \mathrm{~g}$ of Triton X$100,2.29 \mathrm{~mL} n$-heptanol, and $4.32 \mathrm{~mL}$ of cyclohexane. The mixture was mixed for $10 \mathrm{~min}$ followed by adding of $0.2 \mathrm{~mL}$ $\mathrm{NH}_{3}(28-30 \%)$ dissolved in $1.1 \mathrm{~mL}$ of water. The obtained microemulsion was then mixed for $10 \mathrm{~min}$. After that, we have prepared solution 2 by mixing of $2.38 \mathrm{~g}$ of Triton X$100,2.29 \mathrm{~mL}$ of $\mathrm{n}$-heptanol, $9.32 \mathrm{~mL}$ of cyclohexane, $0.2 \mathrm{~mL}$ of TEOS. Next, the solution 2 was added dropwise to the previously prepared microemulsion (adding rate $1 \mathrm{~mL} / \mathrm{min}$ ). After $24 \mathrm{~h}$ of stirring silica nanoparticles were precipitated 
form microemulsion with acetone, centrifuged and washed by ethanol/acetone mixture (1:1), ethanol (2 times) and several times with doubly distilled water.

2.1d Synthesis of SNs-II: $0.016 \mathrm{~g}$ of the synthesized oleate-coated iron-oxide nanoparticles were dispersed in $5 \mathrm{~mL}$ of cyclohexane using ultrasound water bath for $60 \mathrm{~min}$. Then, the obtained dispersion was added dropwise using a syringe pump (adding rate $1 \mathrm{~mL} / \mathrm{min}$ ) to the mixture of $2.38 \mathrm{~g}$ of Triton X-100, $2.29 \mathrm{~mL} n$-heptanol, and $4.32 \mathrm{~mL}$ of cyclohexane. The mixture was stirred for $10 \mathrm{~min}$ followed by adding of $0.2 \mathrm{~mL} \mathrm{NH}_{3}(28-30 \%)$ dissolved in $1.1 \mathrm{~mL}$ of water. The obtained microemulsion was then mixed for $10 \mathrm{~min}$. After that, we have prepared solution 2 by mixing of $2.38 \mathrm{~g}$ of Triton X-100, $2.29 \mathrm{~mL}$ of $n$-heptanol, $9.32 \mathrm{~mL}$ of cyclohexane, $0.2 \mathrm{~mL}$ of TEOS. Next, the solution $\mathbf{2}$ was added dropwise to the previously prepared microemulsion (adding rate $1 \mathrm{~mL} / \mathrm{min})$. After $24 \mathrm{~h}$ of stirring $(750 \mathrm{rpm}) 1.1 \mathrm{~mL}$ of [Gd(TCAS)] water suspension $(7.8 \mathrm{mM})$ was added followed by adding of $0.2 \mathrm{~mL}$ of TEOS. The resulting mixture was stirred for $24 \mathrm{~h}$. The separation and purification of the synthesized nanoparticles was performed as mentioned above.

2.1e Synthesis of SNs-III: $0.016 \mathrm{~g}$ of the synthesized oleate-coated iron-oxide nanoparticles were dispersed in $5 \mathrm{~mL}$ of cyclohexane using ultrasound water bath for $60 \mathrm{~min}$. Then the obtained suspension was added dropwise (adding rate $1 \mathrm{~mL} / \mathrm{min}$ ) with use of syringe pump to the mixture of $2.38 \mathrm{~g}$ of Triton X-100, $2.29 \mathrm{~mL}$ of $n$-heptanol and $4.32 \mathrm{~mL}$ of cyclohexane. In $10 \mathrm{~min}, 1.1 \mathrm{~mL}$ of [Gd(TCAS)] water suspension $(7.8 \mathrm{mM})$ was added. Next, the resulting mixture was stirred for $10 \mathrm{~min}$ again followed by adding of $0.2 \mathrm{~mL}$ of TEOS. The obtained microemulsion was stirred for $10 \mathrm{~min}$. After that, we have prepared solution 2 by mixing of $2.38 \mathrm{~g}$ of Triton X-100, $2.29 \mathrm{~mL}$ of $n$-heptanol, $9.32 \mathrm{~mL}$ of cyclohexane, $0.2 \mathrm{~mL}$ of $\mathrm{NH}_{3}(28-30 \%)$ dissolved in $1.1 \mathrm{~mL}$ of water. Next, solution 2 was added dropwise to the previously prepared microemulsion under an adding rate of $1 \mathrm{~mL} / \mathrm{min}$ and stirred for $24 \mathrm{~h}$ at $750 \mathrm{rpm}$. The separation and purification of the synthesized nanoparticles was performed as mentioned above.

\subsection{Characterization}

\section{2a Inductively coupled plasma optical emission} spectrometry (ICP-OES): $\mathrm{Gd}, \mathrm{Si}$ and Fe were identified in the colloids using simultaneous inductively coupled plasma optical emission spectrometry (ICP-OES) model iCAP 6300 DUO by Varian Thermo Scientific Company equipped with a CID detector. This spectrometer enables the simultaneous measurement of peak heights within the 166-867 nm range. The optical resolution is less than $0.007-200 \mathrm{~nm}$. The working frequency is $27.12 \mathrm{MHz}$. Together, the radial and axial view configurations enable optimal peak height measurements with suppressed spectral noises. The experimentally observed Gd (spectral line-335.047 nm), Fe (spectral line-259.940 nm) and $\mathrm{Si}$ (spectral line-251.611 nm) concentrations are summarized in Table S1 in the Supplementary Information.

2.2b TEM measurements: A certain volume of hexane suspension of iron-oxide nanoparticles (bath-sonicated for about $3 \mathrm{~min}$ prior to TEM analysis) was dispersed on 200 mesh copper grids with continuous formvar support film and hexane was allowed to evaporate. Next, the TEM images were obtained by means of Hitachi HT7700 (Japan) transmission electron microscope at an acceleration voltage of $100 \mathrm{kV}$.

2.2c HRTEM measurements: High-resolution transmission electron microscopy studies (HRTEM) were carried out using FEI Titan 80-300 kV. The microscope operated with an acceleration voltage of $300 \mathrm{kV}$. The iron oxide nanoparticles were gently pressed to disrupt clusters of nanoparticles and form a fine powder. A small amount of the fine powder was then pressed onto a Lacey carbon grid and then imaged. In order to have the nanoparticle arrays on the Lacey carbon grid, the nanoparticles were dispersed in chloroform at a concentration less than $0.5 \mu \mathrm{g} / \mathrm{mL}$ and then bath-sonicated for about $3 \mathrm{~min}$. A small droplet $(3 \mu \mathrm{L})$ of the solution was then drop coated onto the Lacey carbon grid. The grid was left to dry and then imaged.

2.2d FC/ZFC measurements: About $10 \mathrm{mg}$ of ironoxide powder was weighted and the magnetization curve dependence on temperature was measured using SQUID equipment (Quantum Design, Model MPMS-XL, USA). Both FC and ZFC curves were obtained in a temperature range 3$400 \mathrm{~K}$. The FC curve was obtained with an external magnetic field of $100 \mathrm{Oe}$, while the ZFC curve was obtained after the sample was demagnetized (net magnetic field is zero) and cooled to $3 \mathrm{~K}$ prior to the increase in temperature.

2.2e EDX measurements: The EDX measurements were conducted inside the TEM column working at the acceleration voltage of $300 \mathrm{kV}$. The sample was initially tilted $15^{\circ}$ and the EDX signal was collected for $10 \mathrm{~s}$.

2.2f Dynamic light scattering measurements: Dynamic light scattering (DLS) measurements were performed by means of the Malvern Mastersize 2000 particle analyzer. A He-Ne laser operating at $633 \mathrm{~nm}$ wavelength and emitting vertically polarized light was used as a light source. The measured autocorrelation functions were analyzed by Malvern DTS software and the second-order cumulant expansion methods. The effective hydrodynamic radius $\left(R_{H}\right)$ was calculated by the Einstein-Stokes relation from the first cumulant: $\mathrm{D}=\mathrm{k}_{\mathrm{B}} \mathrm{T} / 6 \pi \eta \mathrm{R}_{\mathrm{H}}$, where $\mathrm{D}$ is the diffusion coefficient, $\mathrm{k}_{\mathrm{B}}$ is the Boltzmann constant, $\mathrm{T}$ is the absolute temperature, and $\eta$ is the viscosity. The diffusion coefficient was measured at least three times for each sample. The average error in these experiments is approximately $4 \%$. The samples for DLS study were prepared from deionized water, sonicated for $30 \mathrm{~min}$ and equilibrated at $25.0 \pm 0.1{ }^{\circ} \mathrm{C}$ before DLS and zeta-potential measurements. 

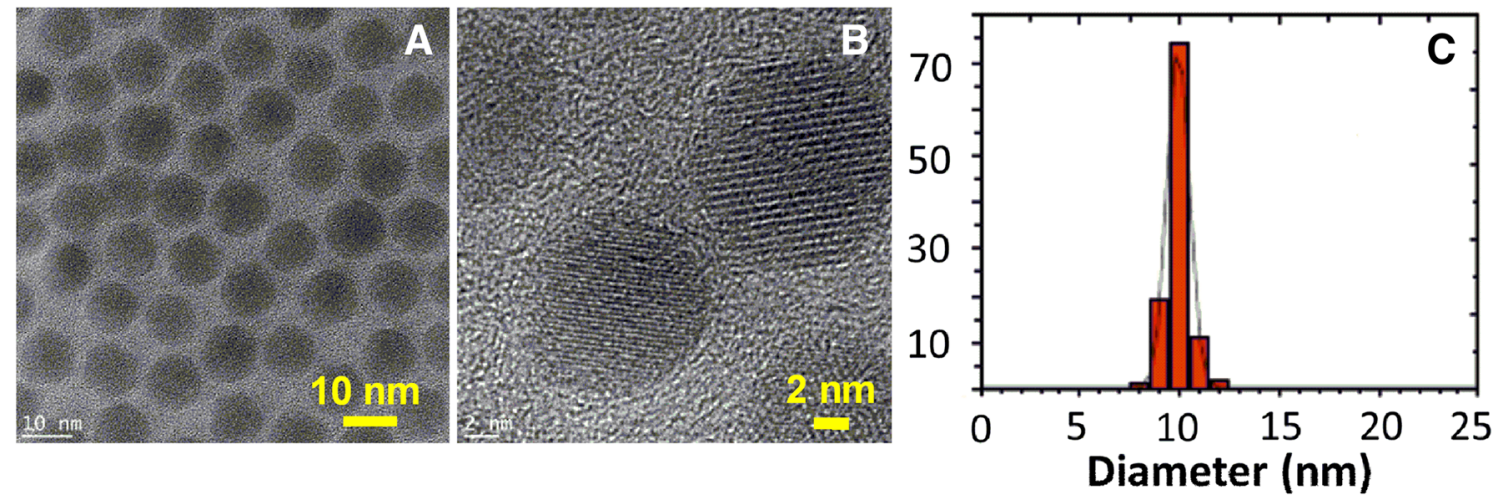

Figure 1. (A) TEM image of the oleate-coated SPIONs, (B) HRTEM image of the oleate-coated SPIONs, (C) Size distribution diagram of the oleate-coated SPIONs.

2.2g pH-metry: $\mathrm{pH}$ of the solutions were measured with Microprocessor $\mathrm{pH}$ meter $« \mathrm{pH} 212 »\left(25^{\circ} \mathrm{C}\right)$ (Hanna Instruments, Germany) The $\mathrm{pH}$-meter was calibrated with standard aqueous buffer solutions (pHs 7.01 and 4.01) $\left(25^{\circ} \mathrm{C}\right.$ ).

2.2h Relaxometric measurements: The prepared samples $(0.5 \mathrm{~mL})$ with different concentrations of the studied colloids were transferred in $10 \mathrm{~mm}$ NMR sample tubes, sonicated using a sonicator water bath and equilibrated at $25.0 \pm 0.1^{\circ} \mathrm{C}$ for $10 \mathrm{~min}$ by use of circulating water bath before relaxometric measurements. The transverse relaxation times $\mathrm{T}_{2}$ of water molecule protons in studied dispersions were measured using the Carr-Purcell-Meiboom-Gill (CPMG) pulse sequence ${ }^{15}$ using a Bruker minispec 20 NMR analyzer (Bruker, Germany). The longitudinal relaxation times $\mathrm{T}_{1}$ of water molecule protons were defined with use of the analyzer's inversion-recovery pulse sequence with 20 data collected for fitting. ${ }^{16}$ The experiments were performed at 19.65 and 59.95 $\mathrm{MHz}$ proton resonance frequency. The relative measurement deviation for transverse and longitudinal relaxation times does not exceed $3 \%$. Transverse $\mathrm{R}_{2}=$ $1 /\left(\mathrm{T}_{2} \mathrm{C}_{\mathrm{Fe}}\right), \mathrm{mM}^{-1} \mathrm{~s}^{-1}$ and longitudinal $\mathrm{R}_{1}=1 /\left(\mathrm{T}_{1} \mathrm{C}_{\mathrm{Gd}}\right)$, $\mathrm{mM}^{-1} \mathrm{~s}^{-1}$ relaxivities were calculated from the measured relaxation times $T_{2}$ and $T_{1}$, respectively; $C_{G d}$ and $C_{F e}$ signify gadolinium and iron concentrations.

High magnetic field vertical bore imaging scanner (Oxford Instruments, Abington, UK) equipped with a Unity/Inova console (Varian, Palo Alto, CA) was used to perform relaxation $\left(\mathrm{T}_{1}, \mathrm{~T}_{2}\right)$ and imaging measurements at $14.1 \mathrm{~T}$. This Varian microimager consists of a vertical wide bore magnet (89 mm) and a $600 \mathrm{MHz}$ Varian Unity/Inova NMR spectrometer equipped with four identical receiver channels and gradient coils with maximum strength of $95 \mathrm{Gauss} / \mathrm{cm}$. The maximum usable magnet diameter is $30 \mathrm{~mm}$. Inversion-recovery and CPMG-pulse sequences were used for longitudinal $\mathrm{T}_{1}$ and transverse $\mathrm{T}_{2}$ relaxation times. A high image quality Spin-Echo-Multi-Slice (SEMS) imaging protocol was applied for $\mathrm{T}_{1}$ - and $\mathrm{T}_{2}$-weighted MRI acquisition. Receiver gain and radiofrequency transmit power was optimized utilizing VnmrJ prescan options. Images were further processed using VnmrJ 1.1D software. A patented 44 tunable transmit/ receive radiofrequency coil was used. ${ }^{17}$

2.2i Cytotoxicity evaluation: The cytotoxicity of the obtained colloids has been determined using a multifunctional system Cytell Cell Imaging (GE Healthcare Life Sciences, Sweden) with Cell Viability BioApp application. The cytotoxicity determination is based on the analysis of fluorescence intensity. ${ }^{18}$

The cell culture WI-38 VA 13 subline 2RA (human embryo lung) form the collection of the Institute of Cytology of Russian Academy of Sciences has been used for cytotoxicity evaluation. The standard nutrient medium 'Igla' with an addition of $10 \%$ of calf embryo serum and $1 \%$ of essential amino acids was applied for cell cultivation.

The cells were dispersed on a panel with 96 holes (cell conc. 100000 cells per $\mathrm{mL}$ ) and cultivated in $\mathrm{CO}_{2}$-incubator at $37^{\circ} \mathrm{C}$. Next, the culture medium was sampled in $24 \mathrm{~h}$ and $150 \mathrm{~mL}$ of the studied colloids were introduced into holes. The experiments were repeated three times.

\section{Results and Discussion}

\subsection{Synthesis and characterization of $10 \mathrm{~nm}$ iron-oxide nanoparticles}

The superparamagnetic oleate-coated iron-oxide nanoparticles were synthesized by high-temperature decomposition of iron oleate precursor in the presence of oleic acid as a stabilizer. ${ }^{12,14}$ Then they were characterized by TEM as $10 \mathrm{~nm}$ spheres (Figure 1, panel A). High-resolution TEM (Figure 1, panel B) indicates that the prepared nanoparticles are single crystals. Besides that, the obtained nanoparticles are uniform-sized as followed form the size distribution diagram (Figure 1, panel C). The zero field cooled (ZFC) and field cooled (FC) measurements showed the blocking temperature of approximately $39 \mathrm{~K}$ implying the superparamagnetic 


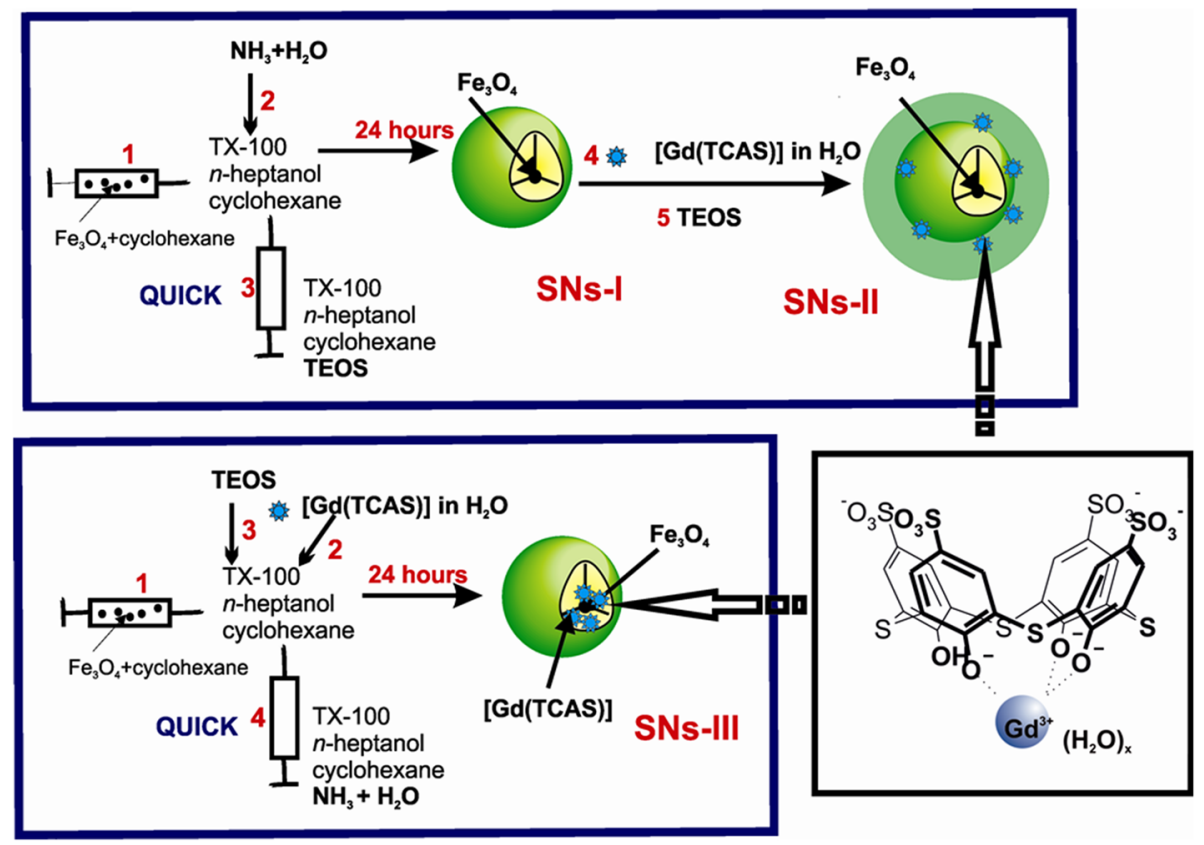

Scheme 1. Schematic presentation of stepwise procedures applied for synthesis of SNs-I, SNs-II, SNs-III.

nature of the nanoparticles at room temperature (Figure 1, panel A). According to XRD analysis, the synthesized nanoparticles consist of pure magnetite phase $\mathrm{Fe}_{3} \mathrm{O}_{4}$ (Figure 1, panel B). The EDX analysis was also performed to prove the purity of the sample (Figure 1, panel $\mathrm{C}$ ). The $\mathrm{Cu}$ peak on EDX pattern is related to the copper grid (Figure 1, panel C).

\subsection{Silica coating of superparamagnetic iron-oxide nanoparticles and doping with [Gd(TCAS)] complexes}

Oleate-coated iron-oxide nanoparticles are almost water-insoluble and prone to aggregation due to hydrophobic oleate shell and, therefore, require surface hydrophilization for their further use in an aqueous medium. Such a hydrophilization can be achieved either by supramolecular self-assembly of water-soluble amphiphiles with oleate chains of iron-oxide nanoparticles or via ligand exchange mechanism as reported by us earlier on $17 \mathrm{~nm}$ SPIONs. ${ }^{19}$ In the present work, in order to impart water-solubility to iron-oxide nanoparticles and avoid their heavy aggregation, we have coated them with a silica layer. The choice of silica is conditioned by its proven biocompatibility, safety, easy functionalization and an ability to dope metal complexes inside its matrix. ${ }^{20}$ Moreover, silica layer provides a protective layer against oxidation and reactive compounds, minimizes leakage of materials embedded inside its matrix and allows the use of nanoparticulate materials in aqueous media. ${ }^{21}$
Firstly, we have synthesized silica-coated iron-oxide nanoparticles (hereafter called SNs-I) by means of well-known microemulsion method which is described in detail in the experimental section and outlined in Scheme $1 .{ }^{22}$

According to coupled plasma, optical emission spectrometry (ICP-OES) data the obtained SNs-I nanoparticles contain both $\mathrm{Si}$ and $\mathrm{Fe}$ in a molar ratio of 1:0.11 (Table S1). The molar ratio of Si, Fe and Gd for SNs-II and SNs-III nanoparticles is 1: 0.06: 0.006 and 1: 0.063: 0.03 , respectively (Table $\mathrm{S} 1$ ).

The TEM analysis revealed the silica-coated ironoxide nanoparticles (SNs-I) with the average diameter of $39 \pm 6 \mathrm{~nm}$ (Figure 2, panel A).

As it can be seen from the TEM image (Figure 2, panel A) some of the SNs-I nanoparticles have one magnetic core, however, some of them include two cores. This experimental finding is explained by probable partial aggregation of hydrophobic oleate-coated nanoparticles during the synthesis when aqueous ammonia is added to the solution. The obtained TEM images also show that iron-oxide cores do not change their size upon silica coating which is a good prerequisite for high transverse relaxivity of the colloids. ${ }^{23}$ According to DLS, the hydrodynamic diameter of SNs-I nanoparticles was measured to be $174.4 \pm 4 \mathrm{~nm}$ with polydispersity index and $\zeta$-potential about 0.3 and $-30 \mathrm{mV}$, respectively (Table 1), which indicates their aggregation at these $\mathrm{pH}$ conditions.

The magnetic relaxation properties of SNs-I-based aqueous colloids have been investigated at different field 

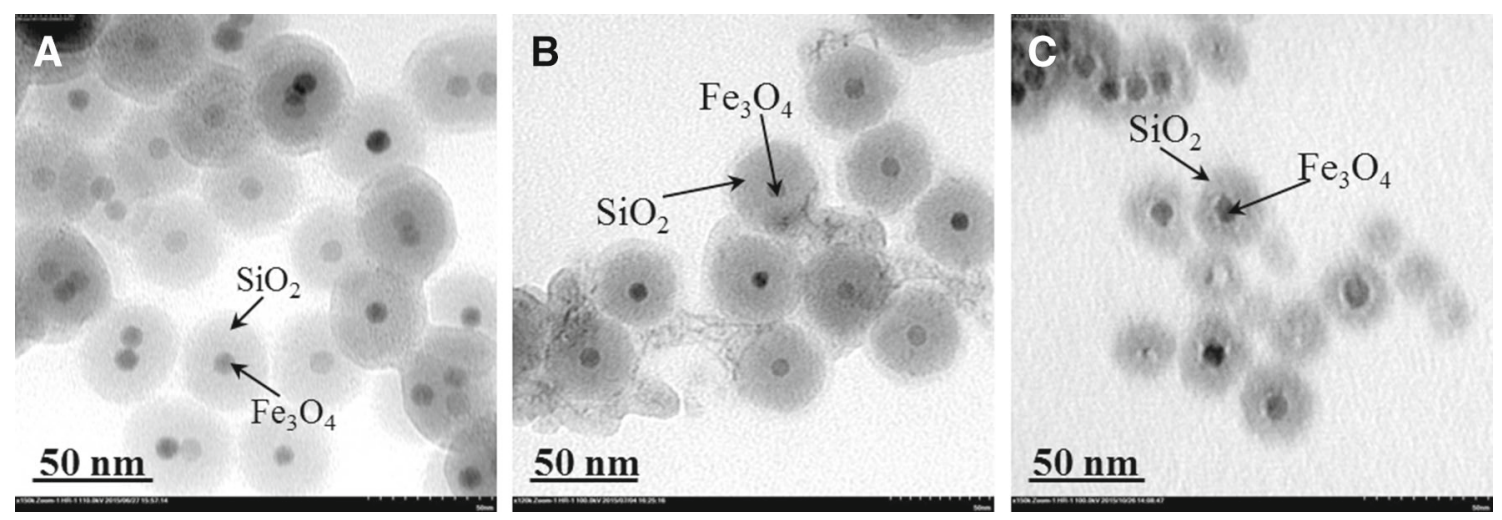

Figure 2. TEM images of SNs: SNs-I (A), SNs-II (B), SNs-III (C), where SNs-I is $\mathrm{SiO}_{2} / \mathrm{Fe}_{3} \mathrm{O}_{4} ; \mathrm{SNs}^{-I I}$ and $\mathrm{SNs}-\mathrm{III}$ are $\mathrm{SiO}_{2} / \mathrm{Fe}_{3} \mathrm{O}_{4} / \mathrm{Gd}(\mathrm{TCAS})$.

Table 1. DLS data of the synthesized $\mathrm{Fe}_{3} \mathrm{O}_{4}$ - and $\mathrm{Fe}_{3} \mathrm{O}_{4}$ [Gd(TCAS)]-doped silica nanoparticles in aqueous solutions $\left(25^{\circ} \mathrm{C}\right)^{\mathrm{a}}$.

\begin{tabular}{lccccc}
\hline Colloid & $\mathrm{C}_{\mathrm{SNs}}, \mathrm{g} \mathrm{L}^{-1}$ & $\mathrm{~d}^{\mathrm{b}}, \mathrm{nm}$ & $\mathrm{PDI}$ & $\mathrm{pH}$ & $\zeta$-potential, mV \\
\hline SNs-I & 0.200 & $174.4 \pm 4$ & 0.30 & 6.78 & $-30.1 \pm 4.0$ \\
SNs-II & 0.433 & $174.4 \pm 4$ & 0.31 & 6.58 & $-32.7 \pm 6.4$ \\
SNs-III & 0.433 & $134.1 \pm 1$ & 0.21 & 6.75 & $-31.1 \pm 5.2$ \\
\hline
\end{tabular}

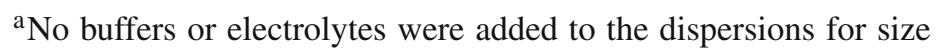
and $\zeta$-potential measurements.

${ }^{\mathrm{b}}$ By intensity.

strengths of $0.47,1.41,14.1 \mathrm{~T}$ (Figure 3, Table 2). As follows from the obtained data, the transverse relaxivity values of SNs-I aqueous colloids at $0.47,1.41$ and 14.1 $\mathrm{T}$ are $134.74,163.11$ and $153.84 \mathrm{mM}^{-1} \mathrm{~s}^{-1}$, respectively (Table 2). Since SNs-I nanoparticles possess high transverse and insignificant longitudinal relaxivity values they may serve only as single-mode contrast agents providing a good darkening effect on the MRI images. In order to prepare nanoparticles with double-mode contrasting one should combine superparamagnetic and paramagnetic entities within one nanoparticle. ${ }^{6}$ As it was shown earlier, the inner-sphere complex of $\mathrm{Gd}(\mathrm{III})$ with $p$-sulfonatothiacalix[4]arene (hereafter called [Gd(TCAS)]) in which Gd(III) ion is also bound with 5 or 6 water molecules is stable ( $\lg \beta \sim 11, \lg \beta$ is the logarithm of the binding constant of the $[\mathrm{Gd}(\mathrm{TCAS})]$ complex) and does not decompose in alkaline solutions. ${ }^{24}$ In addition, according to our previous article, [Gd(TCAS)] complex can be doped into the silica matrix resulting in non-toxic nanoparticles with high longitudinal relaxivity. ${ }^{21}$ It should be added that the silica matrix allows inner and superficial localization of $[\mathrm{Gd}(\mathrm{TCAS})]$ complexes depending on the synthetic conditions. ${ }^{21}$ This, in turn, triggers significant changes in relaxometric properties of the nanoparticles. Besides that, the longitudinal relaxivities of paramagnetic Gd(III)-based nanoparticles is considerably higher than that of aqueous [Gd(TCAS)] complex. In particular, the longitudinal relaxivity of aqueous [Gd(TCAS)] complex is about $4.18 \mathrm{mM}^{-1} \mathrm{~s}^{-1}$, whereas the longitudinal relaxivity of [Gd(TCAS)]-doped silica nanoparticles vary from 4.08 to $49.68 \mathrm{mM}^{-1} \mathrm{~s}^{-1}$ depending on the site of localization of [Gd(TCAS)] within silica matrix. ${ }^{21}$ The high relaxivity of $49.68 \mathrm{mM}^{-1} \mathrm{~s}^{-1}$ is explained by the limited mobility of [Gd(TCAS)] complexes in solid silica shell and better water molecules exchange when [Gd(TCAS)] complexes are localized superficially. ${ }^{21}$ These recent results have prompted us to synthesize nanoparticles with superparamagnetic iron-oxide cores coated with silica shell which is doped with paramagnetic [Gd(TCAS)] complexes. Such nanoparticles may fulfil a double relaxation function in which iron-oxide cores are responsible for shortening of transverse relaxation rates, whereas paramagnetic shell shortens the longitudinal relaxation rates of water protons.

The 'double' nanoparticles bearing superparamagnetic iron-oxide cores and paramagnetic [Gd(TCAS)] complexes were synthesized through microemulsion 

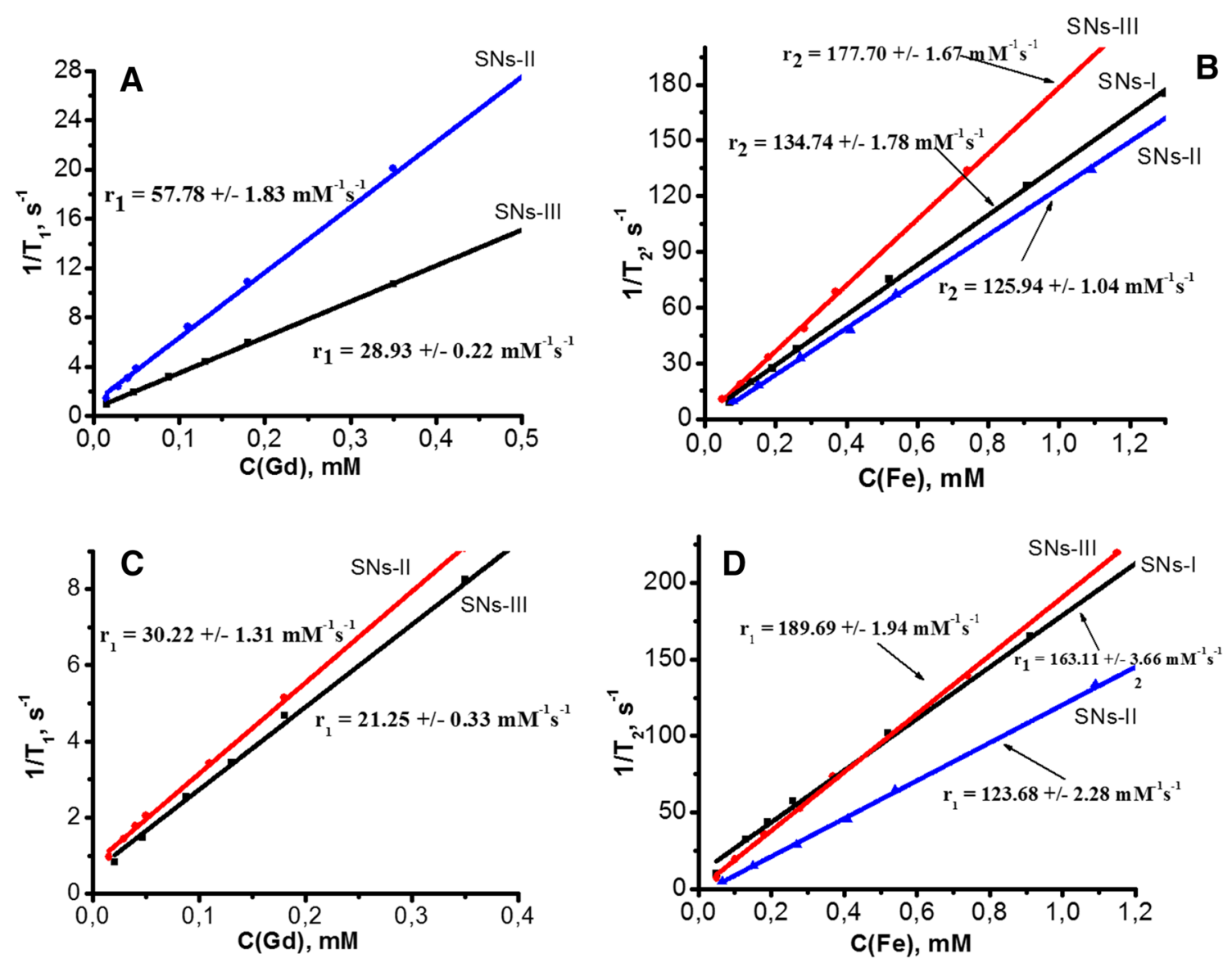

Figure 3. $1 / \mathrm{T}_{2}$ and $1 / \mathrm{T}_{1}$ (panel $\mathrm{B}$ ) versus $\mathrm{Gd}(\mathrm{III})$ and $\mathrm{Fe}$ concentrations for the obtained colloids. $0.47 \mathrm{~T}$ (Panels A and B), 1.41 T (Panels C and D). SNs-1 stands for silica-coated iron-oxide nanoparticles, whereas SNs-II and SNs-III stand for [Gd(TCAS)]-doped silica-coated iron-oxide nanoparticles. The measurements are performed at $25^{\circ} \mathrm{C}$.

method involving alkaline hydrolysis of TEOS and its subsequent polymerization in the presence of iron-oxide nanoparticles and [Gd(TCAS)] complexes (Scheme 1). For the synthesis of SNs-II, iron-oxides was first introduced into the synthetic mixture followed by addition of $[\mathrm{Gd}(\mathrm{TCAS})]$ aqueous dispersion without additional alkalization in $24 \mathrm{~h}$ with subsequent adding of extra TEOS. For the synthesis of SNs-III, iron-oxides and [Gd(TCAS)] aqueous dispersion were introduced into the synthetic mixture almost simultaneously (see the experimental section for details). The conditions for the synthesis of SNs-I, II, III are described in the experimental section.

TEM analysis of SNs-II indicates core-shell spherical, monodisperse nanoparticles with average diameter of $47 \pm 10 \mathrm{~nm}$ with worm-like structures (Figure 2), whereas TEM imaging of SNs-III colloids reveals two types of nanoparticles: spherical core-shell nanoparticles with average diameter of $32 \pm 6$ and silica nanoparticles of $28 \pm 10 \mathrm{~nm}$ diameter with no magnetic core. It must be added that in the case of synthesis of SNs-II and SNs-III the role of "nuclei" may play not only iron-oxides but also undissolved complexes [Gd(TCAS)] leading to the aggravation of TEM images ongoing form SNs-I to SNs-II and SNs-III.

According to DLS the hydrodynamic diameters of SNs-II and SNs-III nanoparticles are about 174.4 \pm 4 and $134.1 \pm 1 \mathrm{~nm}$, respectively (Table 1). Both types of nanoparticles are characterized by similar $\zeta$-potential values of about $-30 \mathrm{mV}$ (Table 1).

The transverse and longitudinal relaxivity values $\left(\mathrm{R}_{2}\right.$ and $R_{1}$, respectively) have been calculated as the slopes of the linear dependencies of water protons relaxation rates versus Gd or Fe concentrations (Figure 3). Relaxation measurements of the obtained colloids have been performed at different magnetic field strengths and summarized in Table 2.

As it can be seen from Table 2, SNs-I nanoparticles demonstrate high transverse and negligible longitudinal relaxivities at $0.47 \mathrm{~T}$. At 1.41 and $14.1 \mathrm{~T}, \mathrm{R}_{2}$ values increase approximately by 21 and $14 \%$, respectively. These nonmonotonic changes of $R_{2}$ upon magnetic 
field increase agree very well with the previous data reported by Palko et al., on the relaxation measurements of different iron-oxides under various magnetic field strengths. ${ }^{2}$

The presence of $\mathrm{Gd}(\mathrm{III})$ ions in the silica matrix of SNs-II nanoparticles gives rise to longitudinal relaxivity of $57.78 \mathrm{mM}^{-1} \mathrm{~s}^{-1}$ at $0.47 \mathrm{~T}$ which is about 12 times higher than that of commercially used Gd-DTPA complex (Magnevist). ${ }^{25}$ Such a high longitudinal relaxivity indicates superficial localization of [Gd(TCAS)] complexes inside silica coating. Nevertheless, the TEM images reveal warm-like silica nanoparticles along with the silica-coated iron oxides, which indicates the formation of silica coated [Gd(TCAS)]-complexes versus their doping into the silica shell of the silica-coated iron oxides. Thus, SNs-II represents two types of differently sized nanoparticles, where the greater sized are encapsulated by the iron oxides, while the smaller sized ones by [Gd(TCAS)]. This morphology results in rather high $\mathrm{R}_{1}$ values, while $R_{2}$ values remain practically unchanged at $0.47 \mathrm{~T}$ (Table 2). The $\mathrm{R}_{2} / \mathrm{R}_{1}$ for SNs-II is about 2.18 which is promising for their potential use as double contrast agents. ${ }^{11}$ The increase of magnetic field strength to $1.41 \mathrm{~T}$ entails significant longitudinal relaxivity to drop from 57.78 to $40.98 \mathrm{mM}^{-1} \mathrm{~s}^{-1}$, whereas transverse relaxivity remains almost the same (Table 2). Besides that, at $1.41 \mathrm{~T}, \mathrm{R}_{2} / \mathrm{R}_{1}$ ratio of SNs-II becomes larger. At $14.1 \mathrm{~T}$ the transverse relaxivity of SNs-II doubly increases to $250 \mathrm{mM}^{-1} \mathrm{~s}^{-1}$, while longitudinal relaxivity is about $30.22 \mathrm{mM}^{-1} \mathrm{~s}^{-1}$ with the $\mathrm{R}_{2} / \mathrm{R}_{1}$ ratio being 8.26.

Another morphology is observed for SNs-III nanoparticles, which results in another relaxation behaviour. The simultaneous addition of both dopants results in the combination in the silica nanoparticles, although the presence of the 'empty' ones indicates that the formation of [Gd(TCAS)]-doped silica nanoparticles is also possible. At $0.47 \mathrm{~T}$ the longitudinal relaxivity was found to be 28.93 , whereas transverse relaxivity has increased to $177.70 \mathrm{mM}^{-1} \mathrm{~s}^{-1}$ with respect to SNs-I. This can be probably assigned to the greater porosity of SNs-III as compared with SNs-I nanoparticles due to the presence of $[\mathrm{Gd}(\mathrm{TCAS})]$ complexes inside the silica shell. The greater porosity accelerates the diffusion rate of water molecules within the silica matrix and ensures a closer approach of water to the magnetic core which, in turn, triggers transverse relaxivity increase. ${ }^{19}$ Upon increasing the magnetic field strength, the relaxivities of SNs-III change steadily but in opposite directions: longitudinal relaxivities fall, whereas transverse relaxivities go up. The longitudinal relaxivity of SNs-III is smaller than that of SNs-II, which can be explained by the effect of the iron oxide-mediated magnetic field on 


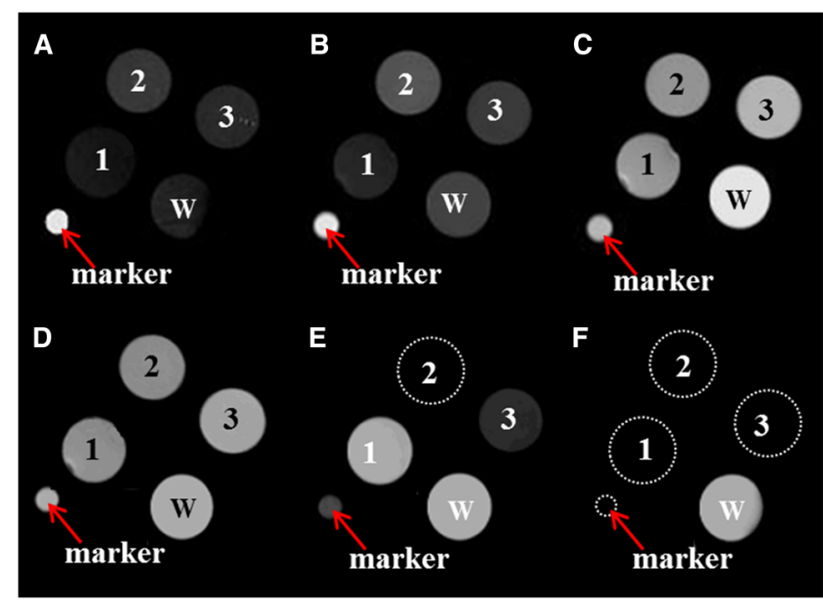

Figure 4. Magnetic resonance images of $\mathrm{T}_{1}-(\mathrm{a}, \mathrm{b}, \mathrm{c})$ and $\mathrm{T}_{2}$-weighted $(\mathrm{d}, \mathrm{e}, \mathrm{f})$ images of NPs I-III $\left(C=0.5 \mathrm{~g} \mathrm{~L}^{-1}\right)$ measured at $14.1 \mathrm{~T}$ for different repetition times (TR) and echo-times (TE): TR $=100 \mathrm{~ms}(\mathrm{a}), \mathrm{TR}=1 \mathrm{~s}(\mathrm{~b}), \mathrm{TR}=10 \mathrm{~s}$ (c), $\mathrm{TE}=10 \mathrm{~ms}(\mathrm{~d}), \mathrm{TE}=50 \mathrm{~ms}(\mathrm{e}), \mathrm{TE}=200 \mathrm{~ms}$ (f).

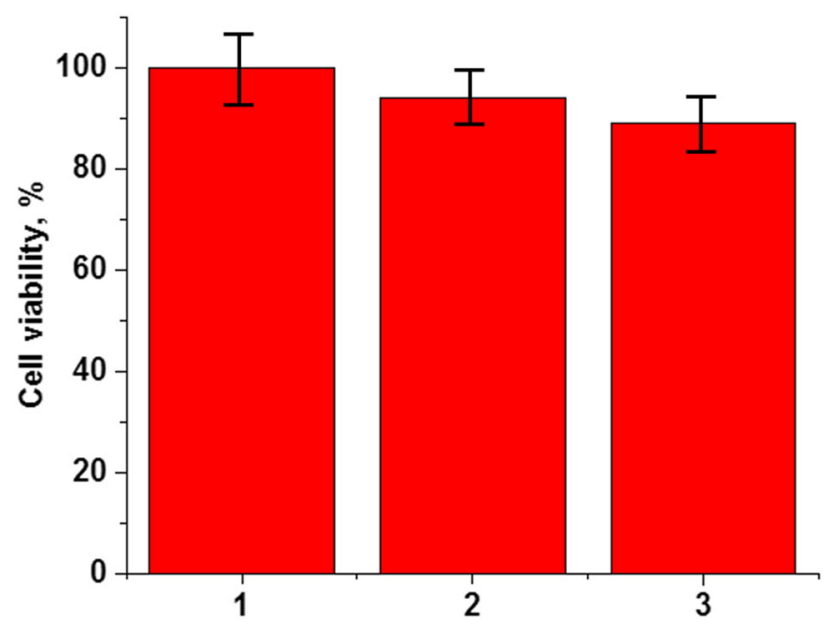

Figure 5. Cell viability test on human embryo lung cells without (1) and incubated by $0.1 \mathrm{mg} \mathrm{mL}^{-1}$ (2) and $0.25 \mathrm{mg} \mathrm{mL}^{-1}$ of SNs-III (3) for $4 \mathrm{~h}$. Error bars denote standard deviations of the cell viability test determined from 3 measurements.

the paramagnetic properties of [Gd(TCAS)] complexes inside the dual nanoparticles.

In order to test the contrasting effect of the synthesized colloids $T_{1}$ - and $T_{2}$-weighted MRI images were obtained at $14.1 \mathrm{~T}$ (Figure 4). As follows from SEMS images, all the nanoparticles show strong darkening and/or brightening effect (Figure 4).

Nanoparticles SNs-III containing superparamagnetic and paramagnetic moieties have demonstrated the best darkening and brightening effect in spite of the fact that their $R_{2} / R_{1}$ is greater than that of SNs-II nanoparticles (Table 2). This makes them prospective candidates for their use as double contrast agents for MRI.

\subsection{Cytotoxicity evaluation of the colloids}

The high $\mathrm{R}_{2}$ and $\mathrm{R}_{1}$ values for SNs-III colloids make them promising material for their further application as double-mode MRI contrast agents. Therefore, the influence of these colloids on cell viability has been determined. The cell viability of the human embryo lung cells was measured in the presence of the prepared SNs-III colloids with a concentration of 0.1 and $0.25 \mathrm{mg} \mathrm{mL}^{-1}$ ensuring high transverse and longitudinal relaxation rates. The cell viability bar charts outlined in Figure 5 show insignificant cytotoxicity of SNs-III colloids which build a basis for their further testing in vivo as prospective dual-mode contrast agents in magnetic resonance imaging.

\section{Conclusions}

Summarizing the data obtained, we have synthesized three different colloids with excellent relaxometric characteristics at different magnetic field strengths. Colloids SNs-I with high transverse relaxivities may function as negative contrast agents under varying magnetic fields. Colloids SNs-III may serve as dual-mode non-toxic contrast agents under different magnetic fields providing good brightening and darkening contrast. Although we have not produced the nanoparticles with enormous $R_{2}$ and $R_{1}$ values and $R_{2} / R_{1}$ ratio close to 1 , we have managed to reveal the correlation between relaxometric behaviour and morphology of the nanoparticles, which, in turn, arise from different ways of synthetic procedures. It must be added that all of the studied colloids demonstrate very good contrasting effect at $1.41 \mathrm{~T}$ which is close to $1.5 \mathrm{~T}$, where most of the medical MRI scanners operate worldwide.

\section{Supplementary Information (SI)}

The Supplementary Information for this article contains FC/ZFC curves, XRD and EDX patterns of the synthesized oleate-coated iron-oxide nanoparticles. SI includes transmission electron microscopy and coupled plasma optical emission spectrometry (ICP-OES) data for SNs-I, SNs-II, SNs-III. Supplementary Information is available at www.ias. ac.in/chemsci.

\section{References}

1. Corti M, Lascialfari A, Marinone M, Masotti A, Micotti E, Orsini F, Ortaggi G, Poletti G, Innocenti C and Sangregorio C 2008 Magnetic and relaxometric properties of polyethyleneimine-coated superparamagnetic MRI contrast agents J. Magn. Magn. Mater. 320 e 316 
2. Jedlovszky-Hajdu A, Tombacz E, Banyai I, Babos M and Palko A 2012 Carboxylated magnetic nanoparticles as MRI contrast agents: Relaxation measurements at different field strengths J. Magn. Magn. Mater. 234 3173

3. Zhang F, Huang X, Qian C, Zhu L, Hida N, Niu G and Chen X 2012 Synergistic enhancement of iron oxide nanoparticle and gadolinium for dual-contrast MRI Biochem. Biophys. Res. Commun. 425886

4. Choi Jin-sil, Lee J-H, Shin T-H, Song H-T, Yeop Kim E and Cheon J 2010 Self-confirming "AND" Logic Nanoparticles for Fault-Free MRI J. Am. Chem. Soc. 132 11015

5. Caravan P 2006 Strategies for increasing the sensitivity of gadolinium based MRI contrast agents Chem. Soc. Rev. 35512

6. Shin T-H, Choi Jin-sil, Yun S, Kim I-S, Song H-T, Kim Y, Park K I and Cheon J $2014 \mathrm{~T}_{1}$ and $\mathrm{T}_{2}$ Dual-Mode MRI Contrast Agent for Enhancing Accuracy by Engineered Nanomaterials ACS Nano 83393

7. Szpak A, Fiejsasz S, Prentoda W, Straczek T, Kapusta C, Szmyd J, Nowakowska M and Zapotoczny S 2014 $\mathrm{T}_{1}-\mathrm{T}_{2}$ Dual-mode MRI contrast agents based on superparamagnetic iron oxide nanoparticles with surface attached gadolinium complexes J. Nanopart. Res. 16 2678

8. Yang M, Gao L, Liu K, Luo C, Wang Y, Yu L, Peng $\mathrm{H}$ and Zhang W 2015 Characterization of $\mathrm{Fe}_{3} \mathrm{O}_{4} / \mathrm{SiO}_{2} / \mathrm{Gd}_{2} \mathrm{O}\left(\mathrm{CO}_{3}\right)_{2}$ core/shell/shell nanoparticles as T1 and T2 dual mode MRI contrast agents Talanta 131661

9. Cai H, An X, Wen S, Li J, Zhang G, Shi X and Shen M 2015 Facile Synthesis of $\mathrm{Gd}(\mathrm{OH})_{3}$-Doped $\mathrm{Fe}_{3} \mathrm{O}_{4}$ Nanoparticles for Dual-Mode $\mathrm{T}_{1}$ - and $\mathrm{T}_{2}$-Weighted Magnetic Resonance Imaging Applications Part. Part. Syst. Charact. 32934

10. Xiao N, Gu W, Wang H, Deng Y, Shi X and Ye L $2014 \mathrm{~T}_{1}-\mathrm{T}_{2}$ dual-modal MRI of brain gliomas using PEGylated Gd-doped iron oxide nanoparticles J. Colloid Interface Sci. $\mathbf{4 1 7} 159$

11. Keasberry N, Banobre-Lopez M, Wood C, Stasiuk J, Gallo J and Long N 2015 Tuning the relaxation rates of dual $\mathrm{T}_{1} / \mathrm{T}_{2}$ nanoparticle contrast agents: a study into the ideal system Nanoscale 716119

12. Bronstein L, Huang X, Retrum J, Schmucker A, Pink M, Stein B and Dragnea B 2007 Influence of Iron Oleate Complex Structure on Iron Oxide Nanoparticle Formation Chem. Mater. 193624

13. Iki N, Fujimoto T and Miyano S 1998 A New WaterSoluble Host Molecule Derived from Thiacalixarene Chem. Lett. 27625

14. Stepanov A, Mustafina A, Mendes R, Rümmeli M, Gemming T, Popova E, Nizameev I and Kadirov M 2016 Impact of heating mode in synthesis of monodisperse iron oxide nanoparticles via oleate decomposition J. Iran Chem. Soc. 13299
15. Meiboom S and Gill D 1958 Modified spin-echo method for measuring nuclear relaxation times Rev. Sci. Instrum. 29688

16. Henoumont C, Laurent S and Elst L Vander 2009 How to perform accurate and reliable measurements of longitudinal and transverse relaxation times of MRI contrast media in aqueous solutions Contrast Media Mol. Imaging 4312

17. Odintsov B 2011 Tunable radio-frequency coil U.S. Patent 8,049,502 B2; November 1

18. Voloshina A, Semenov V, Strobykina A, Kulik N, Krylova E, Zobov V and Reznik V 2017 Synthesis and Antimicrobial and Toxic Properties of Novel 1,3-Bis(alkyl)-6-Methyluracil Derivatives Containing 1,2,3- and 1,2,4-Triazolium Fragments Russ. J. Bioorg. Chem. 43170

19. Stepanov A, Burilov V, Pinus M, Mustafina A, Rümmeli H, Mendes R, Amirov R, Lukashenko S, Zvereva E, Katsuba S, Elistratova J, Nizameev I, Kadirov M and Zairov R 2014 Water transverse relaxation rates in aqueous dispersions of superparamagnetic iron oxide nanoclusters with diverse hydrophilic coating Colloids Surf., A $\mathbf{4 4 3} 450$

20. Nadeem K, Ali L, Gul I, Rizwan S and Mumtaz A 2014 Effect of silica coating on the structural, dielectric, and magnetic properties of maghemite nanoparticles J. NonCryst. Solids $\mathbf{4 0 4} 72$

21. Fedorenko S, Grechkina S, Mustafina A, Kholin K, Stepanov A, Nizameev I, Ismaev I, Kadirov M, Zairov R, Fattakhova A, Amirov R and Soloveva S 2017 Tuning the non-covalent confinement of $\mathrm{Gd}(\mathrm{III})$ complexes in silica nanoparticles for high $\mathrm{T}_{1}$ weighted MR imaging capability Colloids Surf., B 149 243

22. Chen F, Bu W, Chen Y, Fan Y, He Q, Zhu M, Lui X, Zhou L, Zhang S, Peng W and Shi J 2009 A Sub-50-nm Monosized Superparamagnetic $\mathrm{Fe}_{3} \mathrm{O}_{4} @ \mathrm{SiO}_{2} \mathrm{~T}_{2}$-Weighted MRI Contrast Agent: Highly Reproducible Synthesis of Uniform SingleLoaded Core-Shell Nanostructures Chem. Asian J. 4 1809

23. Nadeem K, Ali L, Gul I, Rizwan S and Mumtaz A 2014 Effect of silica coating on the structural, dielectric, and magnetic properties of maghemite nanoparticles J. NonCryst. Solids $\mathbf{4 0 4} 72$

24. Amirov R, McMillan Z, Mustafina A, Chukurova I, Solovieva S, Antipin I and Konovalov A 2005 A first report on ternary complex formation between psulfonatothiacalix[4]arene, tetramethylammonium ion and gadolinium (III) ion in aqueous solutions Inorg. Chem. Commun. 8821

25. Rohrer M, Bauer H, Mintorovitch J, Requardt M and Weinmann H-J 2005 Comparison of Magnetic Properties of MRI Contrast Media Solutions at Different Magnetic Field Strengths Invest. Radiol. 40 715 